IdeAs

Idées d'Amériques

$15 \mid 2020$

Eau et gestion de l'eau dans les Amériques

\title{
De la luzerne aux masterplanned communities : enjeux de la gestion de l'eau sur un front d'urbanisation, le cas de Buckeye en Arizona
}

From growing alfafa to building masterplanned communities: water management issues at the urban fringe, case study of Buckeye, Arizona Desde el cultivo de alfafa hasta la construcción de comunidades planificadas: problemas de expansión urbana y gestión del agua, estudio de caso de Buckeye, Arizona

Anne-Lise Boyer et Rebecca F.A. Bernat

\section{OpenEdition}

Journals

Édition électronique

URL : http://journals.openedition.org/ideas/8272

DOI : $10.4000 /$ ideas.8272

ISSN : 1950-5701

Éditeur

Institut des Amériques

Référence électronique

Anne-Lise Boyer et Rebecca F.A. Bernat, « De la luzerne aux masterplanned communities : enjeux de la gestion de l'eau sur un front d'urbanisation, le cas de Buckeye en Arizona », IdeAs [En ligne], 15 | 2020, mis en ligne le 01 mars 2020, consulté le 25 mars 2020. URL : http://journals.openedition.org/ideas/ 8272 ; DOI : https://doi.org/10.4000/ideas.8272

Ce document a été généré automatiquement le 25 mars 2020.

IdeAs - Idées d'Amériques est mis à disposition selon les termes de la licence Creative Commons Attribution - Pas d'Utilisation Commerciale - Pas de Modification 4.0 International. 


\title{
De la luzerne aux masterplanned communities : enjeux de la gestion de l'eau sur un front d'urbanisation, le cas de Buckeye en Arizona
}

\author{
From growing alfafa to building masterplanned communities: water \\ management issues at the urban fringe, case study of Buckeye, Arizona \\ Desde el cultivo de alfafa hasta la construcción de comunidades planificadas: \\ problemas de expansión urbana y gestión del agua, estudio de caso de Buckeye, \\ Arizona
}

Anne-Lise Boyer et Rebecca F.A. Bernat

\section{Introduction}

Aux États-Unis, d'après un classement des services américains du recensement (U.S. Census) en 2018, les villes dont la population croît le plus vite se situent au sud et à l'ouest, dans les régions les plus chaudes et les plus sèches. Dans le sud-ouest étatsunien semi-aride, la croissance urbaine est même sans commune mesure avec le reste du pays (Liddell E., 2015 ; Nédélec P., 2016; Benites-Gambirazio E. et al., 2016) : en Arizona, la croissance démographique a été de $+783 \%$ sur la période 1950-2010, quand elle a seulement doublé à l'échelle nationale. En 1950, Phoenix, la capitale de l'État, comptait 107000 habitants, en 2010 son aire métropolitaine représente 4,2 millions d'habitants. Cette croissance exponentielle repose notamment sur la dynamique d'étalement urbain, qui vient étendre la superficie de la ville sous une forme peu dense et éparpillée, et fait des zones périurbaines de la région l'un des moteurs de la croissance économique (Le Goix R., 2001 ; Bruegmann R., 2005 ; Benites-Gambirazio E., 2016). 
2 Cette croissance démographique du Sud-Ouest s'accompagne d'une transition économique régionale dans laquelle la grande majorité des nouveaux emplois et revenus est désormais issue du secteur des services, et non plus de l'extraction des ressources, de l'industrie lourde ou de l'agriculture (Gober P., 2006 ; Sheridan T., 2012 ; Boyer A.-L. et al., 2017). Ces changements régionaux impliquent des mutations dans les modes de mise en valeur des ressources naturelles, notamment l'eau (Travis W., 2003), dans un contexte semi-aride où tout développement est conditionné par la disponibilité de cette ressource (Le Tourneau F.M. et F. Dubertret, 2019). Avec l'augmentation de la population, la consommation d'eau glisse du secteur agricole aux usages urbains.

3 Située en bordure ouest de l'agglomération de Phoenix, à $50 \mathrm{~km}$ de la ville-centre, et désignée par l'U.S. Census comme la ville qui croît le plus vite des États-Unis ${ }^{1}$, Buckeye est un cas qui semble faire jouer à l'extrême les dynamiques économiques, sociales, et environnementales qui caractérisent le sud-Ouest états-unien semi-aride. De 726 habitants en 1920, à 6537 en 2000, Buckeye compte aujourd'hui 74370 résidents. L'Association des Gouvernements du comté de Maricopa prévoit même que Buckeye pourrait atteindre une population de plus de 310000 habitants d'ici 2040, soit une augmentation de 326 \% (Matrix Design Group, 2018). À l'origine une bourgade de fermiers fondée à la fin du XIX ${ }^{\mathrm{e}}$ siècle à proximité de la rivière Gila, Buckeye correspond désormais à un front d'urbanisation (Le Goix R., 2002 ; 2016) où les terres agricoles sont de plus en plus converties en terrains constructibles et en lotissements immobiliers.

4 Cependant, ces transformations posent question dans le contexte d'une région marquée depuis une vingtaine d'années par une sécheresse prolongée qui met de plus en plus en jeu l'approvisionnement en eau des centres urbains (Euzen A. et B. Morehouse, 2014 ; Barbier N., 2015 ; Poupeau F. et al., 2016). Ainsi, face à une situation de rareté de la ressource qui va s'aggravant, cette transition d'une zone agricole à une zone urbaine soulève de nombreuses questions et tensions dans la gestion de la ressource à l'échelle locale. Depuis les années 1980, c'est notamment l'exploitation des nappes phréatiques, régulée par le Groundwater Management Act (GMA), qui est au cœur des enjeux. Ainsi, alors que la ville de Buckeye croît à grande vitesse, de plus en plus d'espaces dépendent désormais du Central Arizona Groundwater Replenishment District (CAGRD), dispositif mis en place par l'État d'Arizona dans le but de compenser les pompages excessifs dans les aquifères. Cependant, face à la croissance urbaine frénétique, dominée par les masterplanned communities, des lotissements planifiés par de grands promoteurs, aménagés sur le modèle de l'enclave (comptant logements, commerces, écoles, activités récréatives, etc.) et de l'oasis fortement consommatrice d'eau (cours de golf, piscines, parcs, etc.), la gestion durable de la ressource en eau reste difficile à mettre en œuvre.

5 Ces dynamiques locales s'inscrivent dans le contexte plus large des changements globaux (aridification du climat, changements dans l'occupation des sols, urbanisation, etc.) qui concernent bien d'autres espaces à l'échelle de la planète (Arnauld de Sartre $\mathrm{X} ., 2016$ ) et peuvent être comprises dans le cadre actuel de diffusion du néologisme Anthropocène : une ère géologique dans laquelle les activités humaines modifient irréversiblement leur environnement (Crutzen P., 2002). L'engouement que connait cette notion ainsi que les discussions qu'elle suscite (Bonneuil C. et J.B. Fressoz, 2013) participe du renouvellement de l'approche des problématiques environnementales par les sciences sociales qui portent notamment un intérêt grandissant aux diverses modalités d'action publique ayant pour objectif de résoudre les principaux problèmes environnementaux (Beau R. et C. Larrère, 2018). Sur la question de l'eau, à la suite 
d'auteurs comme Clarke-Sather A. et al. (2017) qui insistent sur l'importance d'examiner à l'échelle locale "les tactiques géopolitiques nouvelles" liées à l'entrée dans l'Anthropocène, et en particulier celles qui articulent eau et droit et mettent en jeu un cadre législatif, nous proposons à travers le cas de Buckeye, d'étudier la dynamique d'étalement urbain et ses conséquences sur les modes de gestion de l'eau dans le cadre de la loi d'Arizona sur la gestion des eaux souterraines, le GMA de 1980.

Le travail présenté ici est issu du croisement de deux projets de recherches doctorales, l'un s'intéressant aux modes de gestion de la rareté de la ressource en eau dans les espaces urbains, l'autre se concentrant sur le fonctionnement du système de recharge artificielle des nappes phréatiques en Arizona. Cet article se fonde sur une analyse de la bibliographie disponible sur la région (publications scientifiques mais aussi littérature grise) et d'entretiens menés auprès des acteurs de l'État (Arizona Department of Water Resources, Central Arizona Project, etc.) et des acteurs municipaux (services des eaux des municipalités de l'agglomération de Phoenix, notamment les hydrologues et les water conservation specialists, en charge de la gestion de la demande municipale). Cette enquête est complétée par des séances d'observation participante dans les instances de décision sur l'eau ouvertes au public (réunion du comité de direction du Central Arizona Project, Plan d'Urgence contre la Sécheresse) et dans des ateliers de gestion de la demande en eau à destination des ménages. C'est dans ce cadre plus large que le cas de Buckeye, mentionné par de nombreux acteurs, a fait l'objet d'une attention plus particulière (collecte de littérature grise, d'articles de presse, d'entretiens d'histoire orale déposés aux archives, visite et entretien au musée local, entretiens avec les services de l'eau, avec la mairie, visites commentées du lotissement Verrado, entretien téléphonique avec le CAGRD). Dans cet article, nous proposons ainsi de revenir sur l'histoire du développement de la ville de Buckeye, conditionné par la disponibilité de la ressource en eau d'abord mise en valeur à des fins agricoles, pour explorer ensuite les contradictions des modalités actuelles d'une gestion désormais urbaine de l'eau qui doit composer avec la croissance urbaine et les projets de développement immobilier d'un côté, et le cadre législatif du GMA de l'autre. 
Figure 1A : Buckeye, Arizona : un front d'urbanisation en bordure ouest de Phoenix

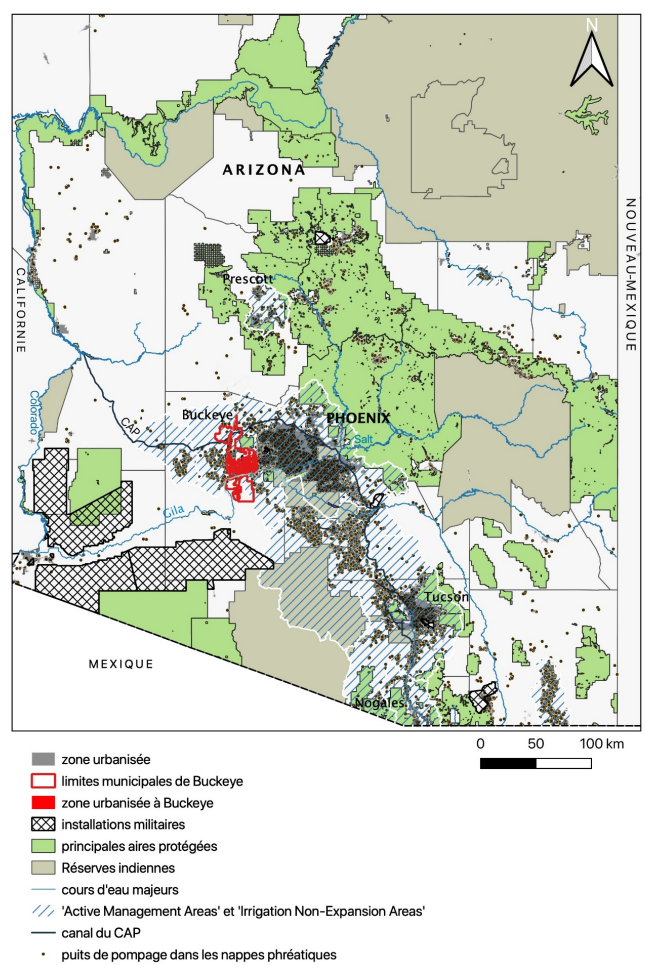

Source : ADWR, University of Arizona GIS, Maricopa County GIS, Google Streets, 2019

Figure 1B : Buckeye, Arizona : un front d'urbanisation en bordure ouest de Phoenix

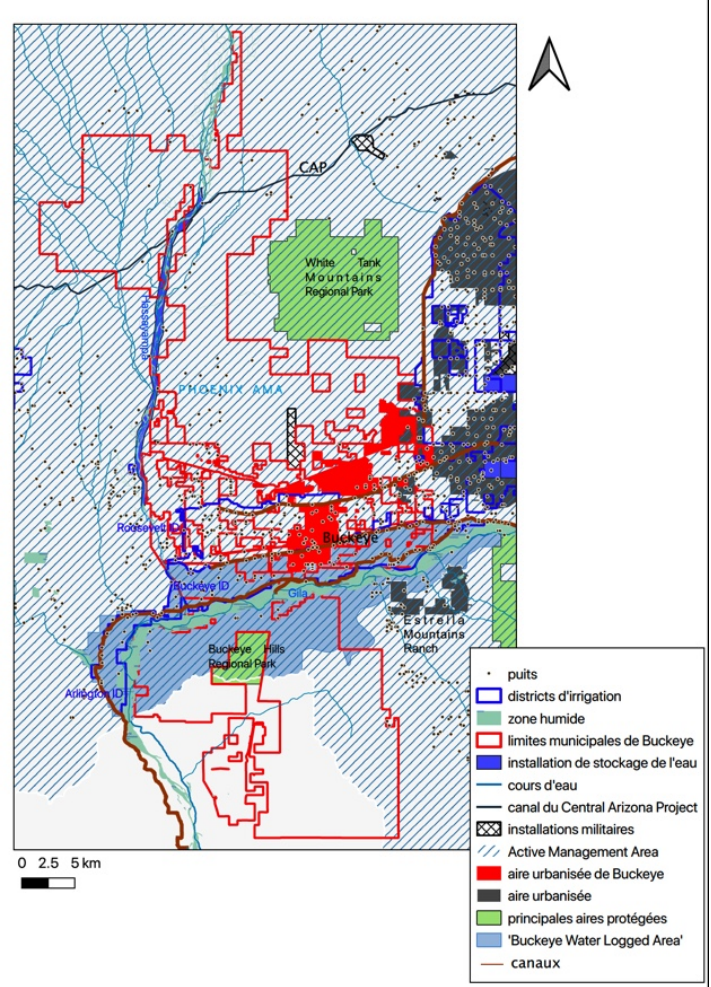

Source : ADWR, University of Arizona GIS, Maricopa County GIS, Google Streets, 2019 


\section{Du désert aux champs de luzerne : la mise en valeur agricole de la vallée de Buckeye}

\section{Éléments de localisation : une vallée semi-aride du désert de Sonora}

7 La vallée de Buckeye se situe dans le désert de Sonora, dans le comté de Maricopa, dans une zone climatique semi-aride où les précipitations sont rares sur de longues périodes. En effet, la moyenne annuelle des précipitations se situe à $200 \mathrm{~mm}$, répartie sur 36 jours de pluie, avec une moyenne mensuelle qui peut atteindre $32 \mathrm{~mm}$ au mois d'août pendant la saison des orages (la «mousson» qui apporte des vagues d'air tropical humide et des orages violents fréquents mais localisés) ou $2 \mathrm{~mm}$ seulement aux mois de mai et juin. La zone connaît aussi des pluies d'hiver, cependant plus faibles qu'en été. La température moyenne sur l'année est de $22^{\circ} \mathrm{C}$ et $90 \%$ des journées sont ensoleillées. Les hivers sont particulièrement doux en comparaison des autres déserts d'Amérique du Nord. En revanche, pendant les trois mois d'été, les températures dépassent quotidiennement $40^{\circ} \mathrm{C}$ et dans les zones de désert, des températures supérieures à $50{ }^{\circ} \mathrm{C}$ ont pu être observées (NOAA, 2018).

La vallée de Buckeye se compose d'une succession de cônes alluviaux provenant des montagnes arides et dénudées des alentours. La rivière Gila, qui coule est-ouest, est le cours d'eau principal qui draine la région, dans laquelle se jettent la rivière Hassayampa et la rivière Agua Fria, toutes deux intermittentes, marquées par une alternance entre tronçons pérenne et éphémère selon les saisons (Photographie 1). Les sols donnent lieu en majorité à des terrains sableux, argileux et limoneux en bordure des cours d'eau. Le développement de l'agriculture remonte à la fin du XIX ${ }^{e}$ siècle, à l'exception de quelques arpents de terres le long des rivières Agua Fria et Gila, cultivés dans le passé par les populations amérindiennes Hohokam qui habitaient la région entre le $\mathrm{III}^{\mathrm{e}}$ siècle et le $\mathrm{XV}^{\mathrm{e}}$ siècle et sont à l'origine des premiers réseaux de canaux (Purdue L., 2015). Après l'abandon du système de canaux Hohokam, le développement agricole s'arrête jusqu'aux années 1860 quand des colons viennent s'installer dans la vallée depuis Phoenix, à $50 \mathrm{~km}$ à l'est, pour cultiver les terrasses alluviales à proximité des rivières dont l'eau peut être facilement divertie pour irriguer les champs. 
Photographie 1 : La rivière Gila, à la confluence avec la rivière Salée et l'Agua Fria, juin 2019

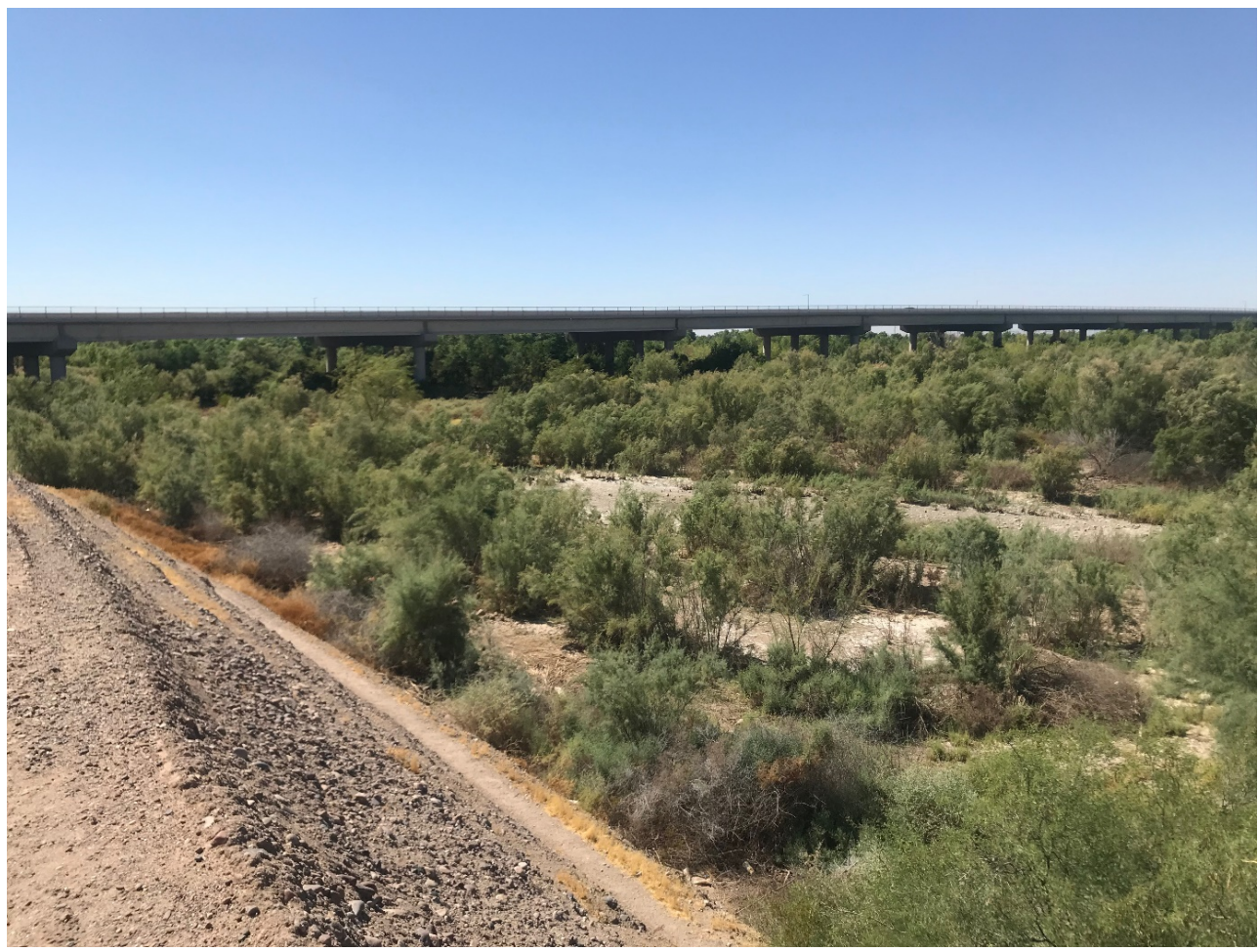

Source : Anne-Lise Boyer

\section{«Premier arrivé, premier servi » : la mise en valeur des eaux de surface grâce au Buckeye Canal}

9 Dans les années 1870 et 1880, dans le contexte de la Conquête de l'Ouest, les politiques économiques et sociales fédérales favorisent le développement par le biais de l'exploitation minière, de l'agriculture et de la création de localités par les colons ${ }^{2}$ (Getches D., 2003). Dans le contexte d'une région semi-aride, c'est notamment l'eau qui va servir de levier principal pour mener à bien la mise en valeur de nouveaux territoires grâce à la Doctrine de l'appropriation antérieure (Doctrine of Prior Appropriation). Initiée lors de la Ruée vers l'or en Californie au milieu du XIX siècle, la doctrine de l'appropriation antérieure détermine une règle simple: ceux qui revendiquent pour la première fois l'accès à l'eau peuvent en faire ce qu'ils veulent (Cech T., 2010). Contrairement à la doctrine riveraine (Riparian Doctrine), qui prévaut à l'est du $100^{\mathrm{e}}$ méridien, nul besoin de s'installer près d'un cours d'eau pour revendiquer un droit sur l'eau, il suffit d'être le premier à construire un canal ou une digue pour acheminer l'eau vers sa propriété. «Premier arrivé, premier servi » devient ainsi la ligne directrice de la gestion de l'eau dans l'Ouest des États-Unis (Miller C., 2000) afin que les individus se trouvent en droit de "s'approprier » l'eau dans le but d'en faire un usage économique et productif (Lasserre F., 2001 ; O’Neill B. et al., 2016).

10 Le centre de l'Arizona connaît d'abord un boom économique dans la vallée de la rivière Salée (Coeurdray M. et al., 2016) qui voit se développer une agriculture intensive irriguée. Avec la mise en exploitation des ressources de la région et l'afflux de population qu'elle génère, se créent les premiers maillages administratifs du territoire :

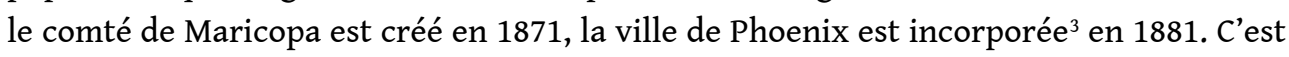


à cette époque que l'économie de l'État d'Arizona s'organise autour des «Cinq $\mathrm{C} »$ historiques : citrus (agrumes), cotton (coton), cattle (bétail), climate (climat), copper (cuivre) (Sheridan T., 2012).

11 Bien vite, la recherche de nouvelles terres agricoles et d'accès à l'eau libre de droit s'étend au-delà de la rivière Salée. En 1884, trois hommes, Malie M. Jackson, Joshua L. Spain et Henry Mitchell, partent de Phoenix et décident de suivre l'ancienne route de fret vers Yuma le long de la rivière Gila, en rive nord, jusqu'à la rivière Agua Fria. Ils sont à la recherche d'un endroit où il serait facile de développer un système d'irrigation. Ils repèrent un point situé à l'ouest de la confluence entre l'Agua Fria et la Gila propice à la construction d'un barrage et d'un canal d'irrigation. Ainsi que le rapporte l'histoire locale ${ }^{4}$, ils marquent l'emplacement sur le tronc d'un saule avec de la poudre à canon avant de retourner à Phoenix enregistrer l'avis légal d'appropriation de la ressource «à des fins agricoles ou hydrauliques " (Parkman I., 1957). En 1886, le Buckeye Canal est construit, appelé ainsi par M. Jackson, en l'honneur de son État d'origine, l'ohio, le «Buckeye State» (l'État du marron). Il marque le début de la transformation de terrains auparavant rugueux et désertiques en des terres agricoles productives (Logan M., 2006). En 1907 est créée la Buckeye Canal Irrigation Company, propriétaire et opérateur du canal qui fournit alors de l'eau depuis la rivière Gila pour l'irrigation de 8000 hectares de terres (Parkman I., 1957).

La première génération d'agriculteurs de la vallée de Buckeye se concentre sur la culture de la luzerne. En effet, celle-ci leur apparaît comme le meilleur mode de mise en valeur agricole de la vallée car elle peut être stockée pour de longues périodes, permet de nourrir le bétail sur place et nécessite moins d'eau que les cultures fruitières, trois facteurs importants à une époque où la vallée est encore isolée. C'est seulement en 1910 que la région de Buckeye est connectée au monde extérieur grâce au chemin de fer qui relie Phoenix à Los Angeles et enfin, dans les années 1920, grâce à l'une des premières autoroutes transcontinentales, l'autoroute 80 qui reliait Savannah en Géorgie à San Diego en Californie (Meck V., 2007).

\section{Toujours plus d'eau : le Roosevelt Irrigation District et les pompages dans la nappe phréatique}

Dans ce contexte de développement rapide, les eaux de surface de la rivière Gila ne paraissent pas suffisantes pour mettre en irrigation de nouvelles terres. La mobilisation des ressources en eaux souterraines s'accroît donc et en 1927, un nouveau canal - le canal Roosevelt - est construit pour récupérer le surplus d'eau du Salt River Project $(\mathrm{SRP})^{5}$, qui, à l'est de Buckeye, doit gérer des problèmes de terrains gorgés d'eau dus aux conditions naturelles de drainage à la confluence Gila River/Salt River, à l'irrigation intensive et aux fuites des canaux (Buckeye Waterlogged Area, voir Figure 1). Pour tenter de résoudre ce problème, le SRP a installé des pompes afin de drainer la zone qui va servir de source d'approvisionnement pour le Roosevelt Irrigation District. Celui-ci est créé dans le but de financer de nouvelles infrastructures pour obtenir cet approvisionnement en eau et mettre en valeur des terres non encore exploitées au nord du canal de Buckeye (Parkman I., 1957). Aujourd'hui, ce sont $78 \%$ des eaux du Roosevelt Irrigation District qui proviennent des nappes phréatiques, pesant ainsi de plus en plus lourdement sur les ressources souterraines. A l'époque, ce projet est un tournant et permet de rendre disponible 14000 hectares supplémentaires 
pour l'agriculture et apporte de nouvelles opportunités dans la vallée : avec ce surplus d'eau, les agriculteurs se tournent vers la culture du coton, dont la production peut désormais être expédiée par voie ferrée ${ }^{6}$. Au début des années 1930, dans le contexte de la Grande Dépression, de nombreux chômeurs vont migrer vers l'Ouest et trouver à travailler dans la culture du coton qui nécessite une main d'œuvre importante (Sheridan T., 2012). En 1929, Buckeye, petite ville prospère passée de 726 habitants en 1920 à 1 077, est incorporée.

14 Aux lendemains de la Seconde Guerre mondiale, la mécanisation croissante du travail agricole réduit le nombre d'ouvriers nécessaires. De plus, à la fin des années 1970, les nouvelles autoroutes 10 (de la Floride à Los Angeles) et 8 (du sud de Phoenix à San Diego) placent Buckeye à un carrefour d'envergure nationale dans un contexte ou l'Arizona s'inscrit pleinement dans la dynamique de la Sun Belt, attirant retraités aisés, appelés snowbirds, qui quittent leur État d'origine pour des régions plus ensoleillées, et populations diplômées intéressées par le développement des secteurs de pointe hérités de l'industrie de l'armement (Ghorra-Gobin C., 1998 ; Benites-Gambirazio E. et al., 2016). Alors que se rendre à Phoenix prend désormais moins d'une heure par l'autoroute 10, la vallée de Buckeye entre dans une nouvelle période de croissance fondée sur l'étalement urbain et le développement immobilier. La vallée traverse ainsi une phase de transition, caractéristique de l'État d'Arizona qui passe d'une période "extractive " autour de la mise en valeur minière et agricole du territoire à une « société urbaine de la Sun Belt » (Sheridan T., 1995 ; 2012) fondée sur le développement immobilier et les services (Ross A., 2011) qui ont pour point commun de nécessiter la mobilisation de fortes quantités d'eau. Dans les années 2000, Buckeye devient le nouveau front d'urbanisation du comté de Maricopa: de nouveaux lotissements s'intercalent désormais entre les exploitations agricoles, de plus en plus reléguées au statut de vestiges du passé agricole de la région (Photographie $1 ; 2$ ). 
Photographie 2 : L'étalement urbain gagne sur les terres agricoles : Cove Dove Estate à Buckeye, juin 2019

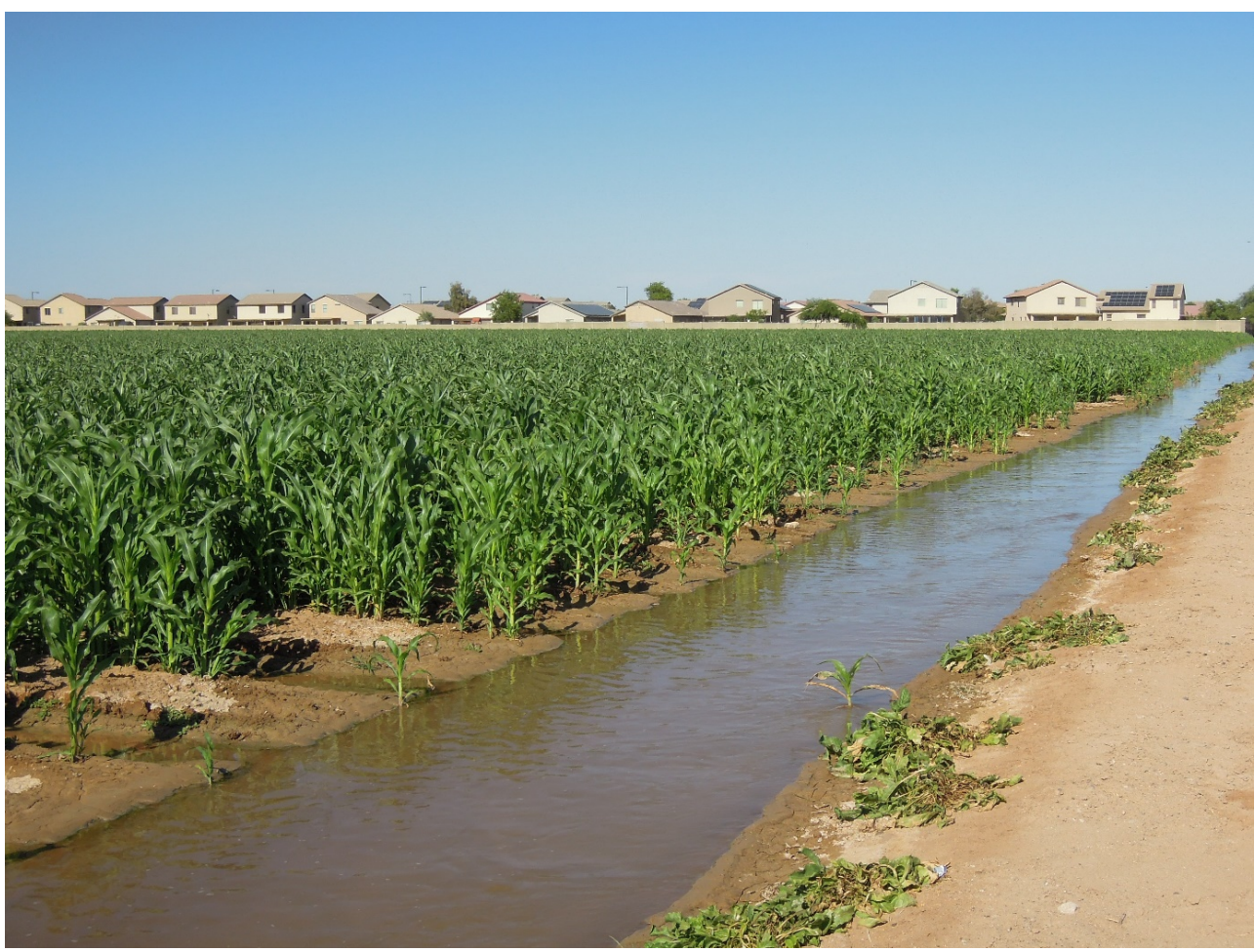

Source : Anne-Lise Boyer

Photographie 3 : Terres agricoles à vendre à Buckeye, Miller Road, juin 2019

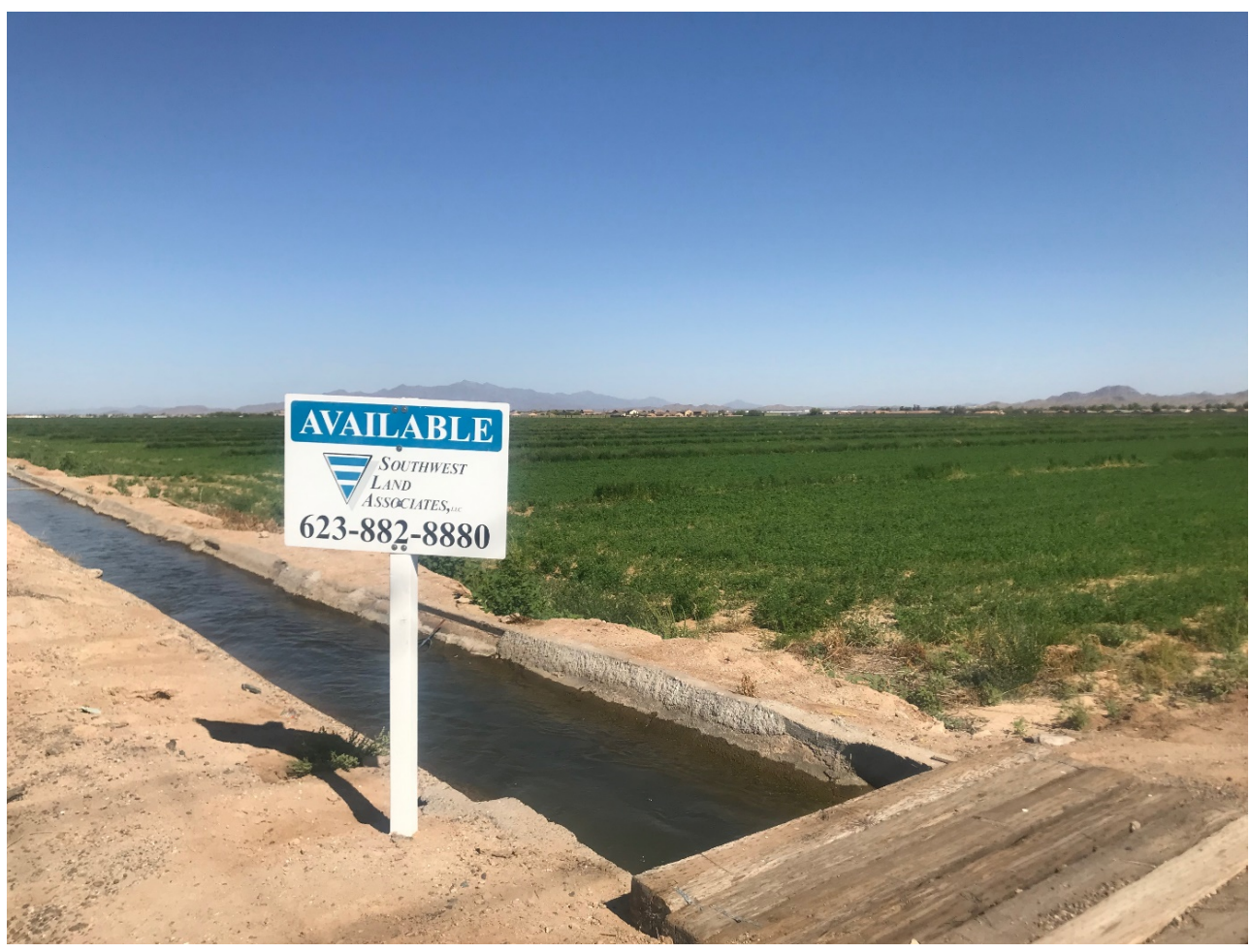

Source : Anne-Lise Boyer 


\title{
Buckeye, un front d'urbanisation en plein boom
}

\author{
Une politique agressive de « land grab » au service de la croissance \\ urbaine
}

15 Même si Buckeye conserve pour l'instant son patrimoine agricole, aujourd'hui, d'après P. Gober (2006), nulle autre ville en Arizona ne montre des stratégies prodéveloppement urbain aussi évidentes. En effet, Buckeye a annexé tellement de terrains en prévision de la croissance urbaine qu'elle est maintenant la plus grande municipalité de la région: d'à peine $3 \mathrm{~km}^{2}$ à sa fondation en 1888 , la municipalité atteint aujourd'hui $1020 \mathrm{~km}^{2}$ dont seulement $8 \%$ sont aménagés (Figure 1). Ce mode d'expansion municipale par annexion est un procédé commun aux États-Unis au XIX siècle. Au Xx $x^{e}$ siècle, il se fait de plus en plus rare sur la côte Est et dans le Midwest mais persiste dans le Sud et dans l'Ouest après la Seconde Guerre Mondiale. En effet, alors que la population s'éloigne des centres-villes et s'installe dans la Sun Belt, l'annexion permet aux municipalités de capter les résidents éloignés et de nouvelles sources de revenus sous la forme de recettes fiscales (Abbott C., 1987). Ainsi certaines municipalités entrent en concurrence voire en conflit au sujet de leurs limites et de l'incorporation de terres adjacentes, ce qui contribue aussi à la mise en place de politiques agressives d'annexion. Dans l'Ouest, les municipalités achètent des parcelles de terre dans le but d'obtenir aussi les droits sur l'eau qui leur sont rattachés afin de sécuriser leur approvisionnement (Barbier N., 2015 ; Benites-Gambirazio E. et al., 2016). C'est surtout dans les années 1990-2000 que Buckeye enregistre une forte augmentation de sa superficie. Par exemple, en 1999, Buckeye a annexé le site qui correspond aujourd'hui au plus grand lotissement planifié de l'aire urbaine de Phoenix, Verrado. Avec 18000 habitants et trois zones commerciales à Verrado, Buckeye peut compter sur des recettes fiscales annuelles de 10 millions de dollars (Heim C., 2008). En 2002, la ville annexe $137 \mathrm{~km}^{2}$ de terres pour développer Douglas Ranch, un projet de masterplanned community financé par JDM Partners, l'entreprise immobilière d'un célèbre homme d'affaires de Phoenix qui a possédé un temps l'équipe de basket Phoenix Suns et l'équipe de baseball Arizona Diamondsbacks. Douglas Ranch est présenté comme un projet de "ville du futur», innovant en termes d'urbanisme et de gestion de l'environnement, construit autour d'un parc inspiré de Central Park à New York en plein désert de Sonora ${ }^{7} .$. Cependant, fortement impactées par la crise de 2008, les premières étapes du projet ne devraient voir le jour qu'en 2020.

16 Aujourd'hui, Buckeye compte 26 masterplanned communities, et 166 lotissements construits par des promoteurs immobiliers, tous sont gérés par des Homeowners Associations (HOAs) ${ }^{8}$, mais sont sous la juridiction de la Ville de Buckeye qui prend en charge les services et infrastructures publics. Ainsi, la municipalité approvisionne en eau $96 \%$ des logements de Buckeye, les $4 \%$ restants sont desservis par des entreprises privées.

\section{Quelle eau pour la croissance urbaine?}

17 Les villes du centre de l'Arizona utilisent différentes ressources en eau : l'eau du fleuve Colorado acheminée par le canal « Central Arizona Project » (CAP), les eaux des rivières à l'intérieur de l'État, les eaux souterraines, ainsi que les eaux usées traitées des 
municipalités (Poupeau F. et al., 2016). Chaque type de ressource est gouverné par une règlementation spécifique (Colby B. et K. Jacobs, 2007). L'eau du Colorado acheminée via le canal CAP est distribuée à certaines municipalités, industriels et nations autochtones possédant des contrats. La Ville de Buckeye par exemple reçoit 83877000 de litres d'eau du CAP par an, mais cela ne correspond qu'à $2 \%$ de l'eau consommée par la ville ${ }^{9}$. Les eaux de surface, comme la rivière Gila ou l'Agua Fria, sont gouvernées par la doctrine de l'appropriation antérieure, et seuls les usagers détenant ces droits sur les eaux de surface peuvent les mobiliser. À Buckeye, ce sont les agriculteurs qui possèdent ces droits.

En 1980, le GMA a créé des zones de gestion appelées Active Management Areas (AMAs) dans lesquelles l'utilisation des eaux souterraines est réglementée par le Département des Ressources en eau de l'Arizona (Arizona Water Resources Department). Il y a désormais cinq AMAs : Phoenix - dans laquelle se situe la ville de Buckeye -, Pinal, Prescott, Santa Cruz et Tucson, qui dans l'ensemble correspondent au couloir de développement urbain nord-sud, formant la région du Sun Corridor ${ }^{10}$. Dans ces zones, il est interdit aux agriculteurs d'étendre l'irrigation à de nouvelles terres, ce qui contribue à renforcer l'expansion urbaine comme nouveau mode de mise en valeur du territoire.

En 1995, des règles appelées « Assured Water Supply (AWS)» (approvisionnement en eau garanti) ont été ajoutées au GMA pour assurer l'approvisionnement en eau des nouveaux développements immobiliers. Pour obtenir un permis de construire au sein d'une AMA, un promoteur immobilier doit donc soit obtenir son propre certificat d'AWS, soit être approvisionné en eau par un service d'eau qui a déjà une appellation AWS. Les règles d'AWS exigent que soit démontrée la disponibilité physique et légale d'une ressource en eau, en continu pendant cent ans, ainsi qu'une capacité financière à traiter et à fournir une eau conforme aux normes de qualité, en cohérence avec l'objectif de gestion de l'eau souterraine de l'AMA et en conformité avec les plans de gestion périodiques requis pour chaque AMA, théoriquement revu tous les dix ans (AAWS Overview, 2019).

Dans l'AMA de Phoenix, l'objectif de gestion de l'eau est de maintenir un «safe-yield ", un équilibre à long terme entre la quantité annuelle d'eau souterraine prélevée et la quantité annuelle de recharge naturelle et artificielle d'ici 2025 : les pompages dans les nappes sont donc fortement limités tant qu'ils ne sont pas pré-rechargés ou remplacés par renflouage artificiel avec de l'eau renouvelable (Bernat R. et al., 2020). En Arizona, les eaux souterraines sont considérées comme non-renouvelables, contrairement aux eaux de surface et aux eaux usées. En effet, dans le Sud-Ouest états-unien, le taux de recharge naturelle des nappes phréatiques est de seulement $8 \%$ et diminuera très certainement de $4 \%$ d'ici 2031-2050 du fait du changement climatique (Niraula R. et al., 2017).

21 Pourtant à Buckeye, les usages résidentiels de l'eau reposent à $98 \%$ sur la mobilisation des ressources des nappes phréatiques. La disponibilité de cette ressource est même mise en avant dans les campagnes de marketing territorial de la municipalité comme le montre cet extrait du livre "City of Buckeye-Welcome to Arizona's biggest opportunity", publié en 2015: "voici les raisons pour lesquelles les nouveaux habitants, entreprises et visiteurs potentiels doivent considérer Buckeye comme l'endroit où s'installer. Vous pouvez construire, nous avons des terrains disponibles. Nous avons de l'eau, Buckeye se trouve sur une nappe phréatique (... $)^{11} »$. 
Cependant, Buckeye n'a pas de certificat d'AWS, bien que la ville ait candidaté en 2008. Dans le but de diversifier son approvisionnement, notamment en ressources renouvelables, et pour prétendre au certificat d'AWS en 2017, la municipalité de Buckeye, avec l'aide d'une entreprise de conseil en ingénierie environnementale, a élaboré un plan directeur de gestion de la ressource en eau ("water master plan») (Carollo Engineers, 2017). D'après ce plan, les solutions qui s'offrent à la Ville sont : acquérir plus d'eau en provenance du CAP, stocker à Buckeye de l'eau du CAP appartenant à d'autres municipalités pour obtenir des crédits de long terme qui permettent de mieux se positionner sur le marché de l'eau, mobiliser des eaux souterraines au-delà de l'AMA de Phoenix dont l'utilisation échappe au GMA (cette dernière option pose cependant la question du coût des infrastructures pour transporter l'eau jusqu'à Buckeye). Par ailleurs, la Ville profite des héritages liés à la mise en valeur agricole de la ressource en eau. Elle a notamment établi un contrat avec l'entreprise privée Buckeye Canal Irrigation Company qui lui fournit notamment l'eau nécessaire à l'irrigation des espaces publics (parcs, terrains de sport, cours d'école, etc.). La majorité du centre-ville de Buckeye, installé sur d'anciennes terres agricoles auxquelles sont attachées un droit à l'eau, ont accès à une pratique héritée de la gestion agricole de la ressource : l'irrigation par submersion («flood irrigation »). Il s'agit d'eau de surface non traitée, délivrée par gravité depuis les canaux gérés par les districts d'irrigation, Buckeye Canal et Roosevelt, vers un système de fossés ouverts, de conduites souterraines, de portes de contrôle et de vannes organisé à l'échelle du quartier. De faible coût et délivré deux fois par mois, cet apport d'eau permet notamment de maintenir des pelouses ou des arbres fruitiers dans le climat semi-aride de la région (Wentz E. et P. Gober, 2007).

Cet affichage d'abondance ne va pas sans poser question au regard de la vitesse à laquelle croit la ville de Buckeye, sur un modèle d'étalement urbain considéré aujourd'hui comme prédateur de ressources naturelles (Bruegmann R., 2005 ; Berque A. et al., 2006) et dans le contexte de changement climatique qui aggrave les pressions sur la ressource en eau à l'échelle régionale. En effet, dans le sud-ouest des États-Unis, les modèles climatiques prévoient une hausse des températures de $2,2^{\circ} \mathrm{C}$ d'ici 2100 qui aurait notamment pour conséquence une réduction du manteau neigeux dans les Rocheuses et une diminution des débits des cours d'eau, et qui pourrait faire entrer la région dans une période de méga-sécheresse de plusieurs décennies (Cayan D. et al., 2013 ; Udall B. et J. Overpeck, 2017).

\section{Les tensions autour de la ressource en eau}

\section{Soutenir la croissance sur le long-terme : le transfert des usages agricoles de l'eau aux usages urbains}

Depuis le GMA en 1980, à l'échelle locale, la concurrence entre les utilisateurs d'eau se fait de plus en plus forte. En effet, le GMA suppose que les usages agricoles de l'eau vont progressivement céder le pas aux usages urbains, à plus haute valeur ajoutée (Blomquist W. et al., 2004). Les acteurs en faveur des usages urbains de l'eau affirment qu'il semble plus intéressant d'affecter une plus grande quantité de la ressource aux grandes zones urbaines, où vivent désormais $80 \%$ de la population de l'État car les villes disposent de moyens financiers plus importants pour financer les coûts de 
l'approvisionnement en eau (Kenski H., 1988). Pourtant, le secteur agricole utilise encore $74 \%$ du total de l'eau consommée en Arizona. Des études ont montré que si les usages agricoles étaient remplacés en intégralité par des usages résidentiels, et que la consommation domestique restait de 750 litres par personne par jour - ce qui est la moyenne dans l'agglomération de Phoenix aujourd'hui (la moyenne nationale s'élève à 315 litres par personne et par jour) - la métropole pourrait accueillir une population urbaine de 9 millions d'habitants (Wright B., 1997 ; Gammage G., 2016). Les opposants à cette idée soutiennent que ce transfert de la ressource aura un impact profond sur les économies rurales locales qui ont contribué à façonner l'attractivité de l'Arizona autour d'un "western ranching lifestyle " (habitat peu dense, centré sur l'extérieur et ouvert sur les grands espaces, inspiré des ranches) (Gammage G. et al., 2011 ; Benites-Gambirazio E. et al., 2016).

La plupart des anciennes familles d'agriculteurs de Buckeye se montrent favorables à la croissance urbaine, conscientes que « désormais, plutôt que la luzerne, le coton ou les produits laitiers, faire pousser des lotissements est devenu la culture commerciale rentable de la région ${ }^{12}$ » (Meck V., 2007). Un urbaniste qui travaille pour la Ville s'en étonne même: "Quand je suis arrivé ici je pensais que ces gens ne voudraient rien changer, qu'ils ne voudraient pas que quiconque touche à leurs terres, mais non, on est complètement dans la situation inverse. Les agriculteurs veulent gagner de l'argent. Dans le contexte actuel: leurs enfants ne veulent pas forcément reprendre l'exploitation, des promoteurs sont intéressés, donc ils veulent vendre... Et certains ne veulent pas refuser 30 millions de dollars ${ }^{13} »$. De plus, il est plus facile de construire sur d'anciennes terres agricoles, car elles ont été déjà aplanies et possèdent bien souvent un accès à l'eau, voire des droits sur l'eau associés à la terre, ce qui réduit le coût des investissements en termes d'infrastructures et de négociations pour démontrer la viabilité de l'approvisionnement sur cent ans. L'État d'Arizona encourage fortement la croissance urbaine grâce à un encadrement minimal de la construction résidentielle (Benites-Gambirazio E., 2016) : tout projet de construction est autorisé sur un terrain de $4000 \mathrm{~m}^{2}$ minimum. Ainsi, les municipalités n'ont pas les compétences pour refuser des projets de développement. À Buckeye, des voix s'élèvent parmi les habitants pour préserver le patrimoine agricole de la ville qui trouvent écho auprès de certains membres du conseil municipal. Pour rester dans le cadre des régulations prévues par l'État d'Arizona, la Ville de Buckeye, dans son Plan Général d'Urbanisme, a défini un zonage spécifique pour des parcelles dédiées à l'agriculture qu'elle souhaiterait laisser en l'état pour préserver un «environnement rural agréable $»^{14}$ qui a un rôle dans l'identité et l'attractivité de la commune. Bien que légalement la Ville ne puisse refuser un projet, le but est de décourager les promoteurs qui souhaiteraient proposer de l'habitat à forte densité (lotissements de petits immeubles à deux étages, ou de maisons mitoyennes par exemple) dans ces zones marquées comme agricoles.

\section{Développement dans le désert et gestion de l'eau à travers le Central Arizona Groundwater Replenishment District (CAGRD)}

Si le transfert de la ressource du secteur agricole aux usages urbains est considéré comme un moyen d'augmenter les sources d'approvisionnement en eau, une part importante de l'expansion urbaine gagne aussi sur des espaces de désert encore vierges de tout développement (Gober P., 2006) - c'est le cas de Douglas Ranch - ou sur des 
terrains désertiques transformés, comme dans le cas de Verrado, construit sur des anciens terrains d'essai Caterpillar au pied des White Tank Mountains.

Cependant, dans ce cas, le développement repose sur l'exploitation des nappes phréatiques, encadrée par la règle d'Assured Water Supply. Dans l'AMA de Phoenix, un lotissement souhaitant obtenir un certificat d'Assured Water Supply ne peut prétendre mobiliser qu'une quantité limitée d'eaux souterraines, correspondant à $2 \%$ de sa demande annuelle en eau escomptée. La garantie de l'approvisionnement sur cent ans doit donc être complétée par l'acquisition de droits sur d'autres ressources en eau ${ }^{15}$. Au-delà de ces $2 \%$, la quantité d'eau souterraine utilisée par le lotissement est considérée comme étant « excessive ». De ce fait, la législature de l'État de l'Arizona a créé le Central Arizona Groundwater Replenishment District (CAGRD) en 1993, une entité gouvernementale qui fournit un mécanisme aux propriétaires fonciers et aux fournisseurs d'eau pour se conformer aux objectifs de gestion durable de la ressource de l'AMA (Megdal S., 2007). Le CAGRD, en centralisant les déclarations de consommations excessives d'eau et en organisant le renflouement des nappes phréatiques, permet donc de mettre en œuvre l'une des règles de l'AWS qui oblige à utiliser des eaux renouvelables (Bernat R. et al., 2020). La création du CAGRD était donc un compromis indispensable pour le passage des règles de l'AWS au niveau législatif, qui, seules, auraient considérablement limité le développement économique dans les AMAs.

28 Un lotissement qui ne dispose pas d'un accès suffisant à une source d'eau renouvelable peut devenir membre du CAGRD (« member land»), ce qui lui permet de se conformer à l'objectif de gestion de l'AMA et donc d'obtenir le certificat d' "Assured Water Supply " dont dépend son existence (Figure 2). Pour bénéficier de ce service, les lotissements dans l'AMA de Phoenix puisant en excès dans les nappes doivent devenir membres du District et s'acquitter de frais d'inscription de 1080 dollars, chaque logement doit aussi payer des frais d'adhésion annuels (28.59 dollars) et une redevance annuelle de 0.59 dollar par mètre cube (CAGRD, 2019). Le CAGRD détermine le coût total du réapprovisionnement des nappes dans chaque AMA et divise ce total par le volume qu'il est obligé de renflouer dans les nappes, il en résulte un taux de renflouement qui est imputé à chaque membre en fonction du volume d'eau souterraine qu'il a utilisé. Les frais de renflouement de l'AMA de Phoenix incluent les coûts d'achat de ressources renouvelables (CAP, effluents, crédits issus de la recharge artificielle), les frais administratifs et la construction d'infrastructures. Le CAGRD a trois ans pour renflouer dans l'AMA de Phoenix l'excès d'eau souterraine pompée pour les besoins du développement urbain.

Une municipalité ou un service d'approvisionnement en eau peut également faire appel au CAGRD (en tant que " member service area ») pour obtenir une appellation d'AWS. Puisque la municipalité de Buckeye n'a pas de désignation d'AWS, les promoteurs immobiliers de 166 lotissements ont rejoint le CAGRD pour obtenir leur propre certificat d'AWS, ce qui oblige ensuite la Ville à les desservir. À l'échelle locale, cela pose la question de la faiblesse d'un pouvoir public limité (en termes de ressources humaines et de budget) sur un territoire municipal immense, à bâtir, ouvert aux investissements immobiliers privés. Par exemple, le service municipal de Buckeye ne compte pour l'instant que 80 employés et se dit parfois débordé ${ }^{16}$.

30 Si le CAGRD permet bien de contrebalancer les quantités d'eau souterraine pompées par ses membres qui dépassent les limites réglementaires, le dispositif est cependant 
sujet à controverse. D'abord, du fait de l'étalement urbain, le CAGRD a vu son nombre d'adhérents fortement augmenter (Avery C. et al., 2007). Selon des estimations menées en 2014, le CAGRD devra obtenir une augmentation de $194 \%$ d'eau disponible pour renflouer l'excès d'eau souterraine pompée par ses adhérents dans les cent prochaines années. Ainsi, la quantité d'eau renouvelable que le CAGRD devra trouver disponible pour recharger les nappes phréatiques ne fait que croître. Dans ce contexte, le CAGRD a prévu des hausses régulières de la redevance liée au renflouement des aquifères (par exemple, 0.74 dollar par mètre cube pour l'année 2024). Ainsi, pour Buckeye, le coût de la participation au système de recharge encadré par le CAGRD pourrait finir par s'élever à 47,2 millions de dollars en 2040, pour environ 300000 habitants (Ferris K. et S. Porter, 2019). À plus long terme, il a été calculé que, si la Ville de Buckeye réussit à obtenir son certificat d'AWS et à rejoindre le CAGRD, et si tout le territoire municipal finit par être aménagé pour des usages résidentiels (il abriterait alors 800000 habitants environ selon Matrix Design Group, 2018), le CAGRD se trouverait en capacité de ne répondre, en termes de recharge, qu'à seulement $20 \%$ de la demande en eau de la ville. Enfin, le système instauré par le CAGRD implique une dépendance quasi totale à l'eau du CAP. Jusqu'en 2004, le CAGRD n'utilisait que l'eau du CAP (Jacobs K. et J. Holway, 2004) pour s'acquitter de ses obligations en matière de renflouement des nappes phréatiques, plus récemment il a pris la décision de diversifier ses approvisionnements en eau, notamment grâce à l'achat d'effluents et de crédits issus de la recharge artificielle (en eau renouvelable) des aquifères (CAGRD, 2015 ; Bernat R. et al., 2020) (Figure 2). En effet, dans le contexte de sécheresse prolongée que connaît la région depuis une quinzaine d'années, le CAP, qui apparaissait jusque-là comme une source d'approvisionnement garantie, montre de plus en plus ses limites. Le Drought Contingency Plan (Plan d'urgence face à la sécheresse), ratifié en mai 2019 par les trois États du bassin du Bas Colorado (Arizona, Californie, Nevada) et l'État fédéral, prévoit une réduction de $6,9 \%$ de l'allocation en eau du Colorado pour l'Arizona afin de conserver une plus grande quantité d'eau dans le lac Mead, le réservoir principal. Cette réduction ne devrait pas toucher directement les usagers mais concerne précisément l'eau destinée au renflouement des nappes phréatiques par le CAGRD. Cet accord vise à repousser l'imminence d'une déclaration de pénurie sur le Colorado (si le niveau du lac Mead passe sous les 325 mètres) qui annulerait totalement la disponibilité de l'eau du CAP pour le CAGRD ${ }^{17}$. Ainsi, le CAGRD est un mécanisme qui permet aux promoteurs immobiliers de se décharger de leur responsabilité envers l'objectif de gestion durable de l'AMA. Devant les difficultés à diversifier l'approvisionnement en eau dans des zones encore peu développées, l'adhésion au CAGRD est même devenue la stratégie par défaut pour les périphéries en croissance des métropoles d'Arizona, Phoenix mais aussi Tucson (Ferris K. et S. Porter, 2019). 
Figure 2 : Groundwater Management Act et étalement urbain : un jeu d'acteurs pour compromis

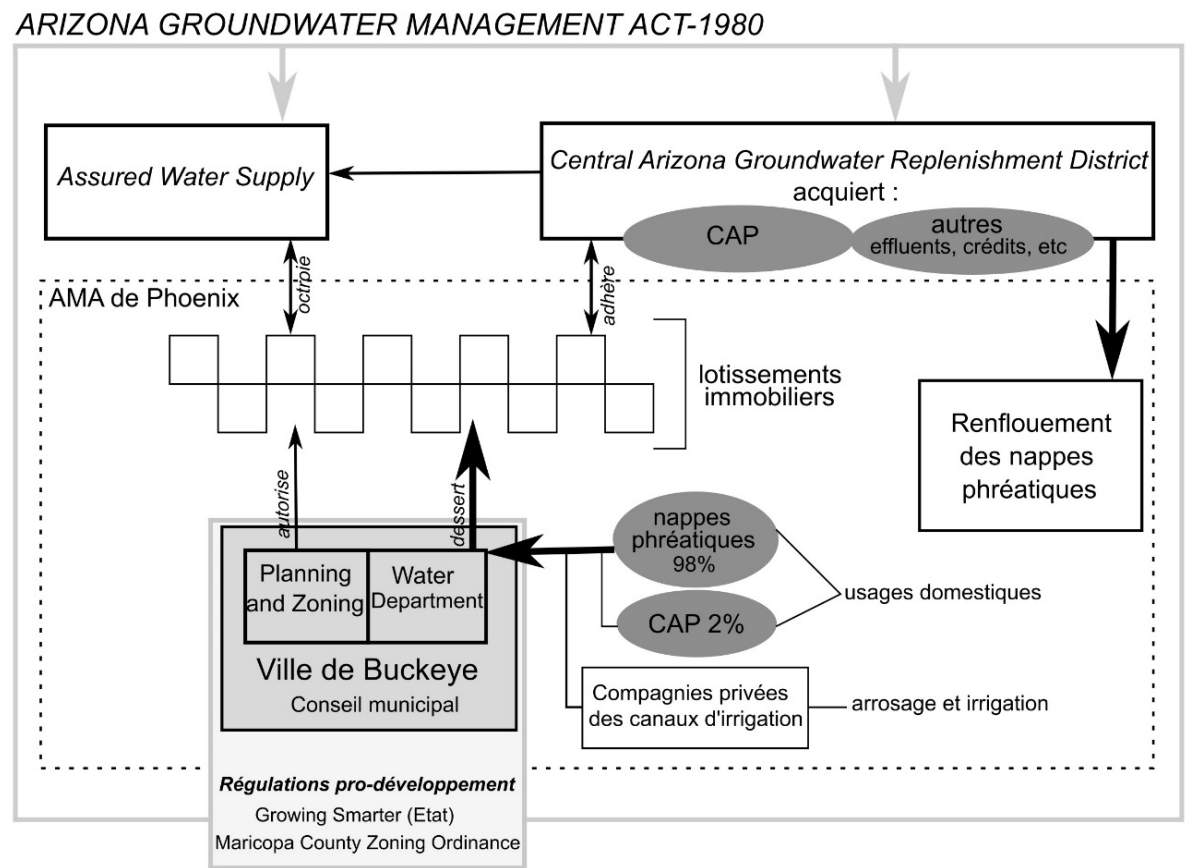

\section{Gérer la demande dans une ville fragmentée, développée par des intérêts privés}

Le développement urbain récent prend place dans de petites municipalités, à l'ouest de Phoenix - comme c'est le cas à Buckeye - qui ne disposent pas des mêmes ressources en eau, plus durables et plus flexibles (accès au CAP, accès au SRP) que les grandes villes de l'aire urbaine de Phoenix (Mesa, Tempe, Scottsdale). Buckeye, malgré les efforts de planification déployés au sein de son "water master plan », a bien du mal à montrer comment elle parviendra, dans le cadre du Groundwater Management Act, de l'Assured Water Supply, et du CAGRD, à fournir de l'eau pour répondre à la demande d'une population escomptée de 800000 habitants.

De plus, l'expansion urbaine de Buckeye se fait au gré des investissements immobiliers, de manière fragmentée. La municipalité de Buckeye s'organise sous la forme d'un archipel de lotissements sans continuité dans le tissu urbain. Ce développement est marqué par le «desert oasis lifestyle » qui favorise l'habitat individuel, peu dense, entrecoupé des paysages verdoyants (pelouses, palmiers) et d'équipements fortement consommateurs d'eau (piscines, golfs) (Hirt P. et al., 2017) (Photographie 4). 
Photographie 4 : Le " desert oasis lifestyle » : Verrado Town Square dans la masterplanned community de Verrado, juillet 2019

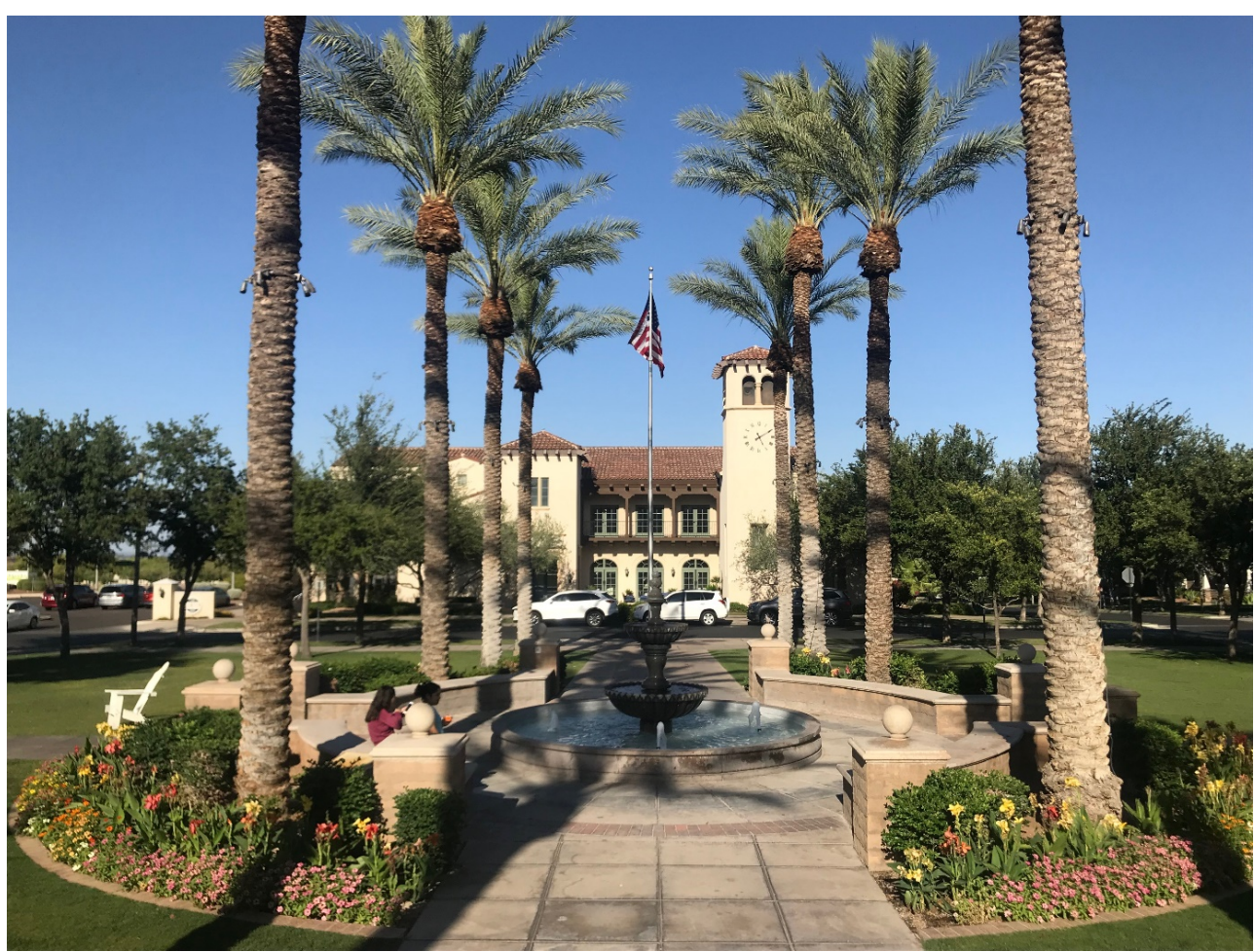

Source : Anne-Lise Boyer

Dans le cadre du GMA, qui promeut la diversification et la sécurisation de l'approvisionnement en eau mais aussi une meilleure gestion de la demande, depuis 2009, la Ville de Buckeye met en place des politiques publiques qui poussent les usagers à contrôler leur consommation. Cependant, l'équipe en charge de proposer et de mettre en œuvre ces mesures n'est constituée pour le moment que de deux personnes.

Pour les usages résidentiels, la tarification se fait selon un système à cinq niveaux : les usagers paient un prix différent en fonction de la quantité dépensée, un prix plus élevé étant facturé pour une quantité plus importante. À Buckeye, $69,5 \%$ de la population se situent dans la première tranche qui consomme entre 0 et $23 \mathrm{~m}^{3}$ d'eau par mois et tout de même 2,1\% de la population consomment plus de $113 \mathrm{~m}^{3}$ par mois (les ménages français utilisent $120 \mathrm{~m}^{3}$ d'eau par an en moyenne) ${ }^{18}$. Dans le contexte semi-aride de la région, entre 50 et $70 \%$ de la consommation d'eau des ménages sont consacrés à des usages extérieurs (Gammage G. et al., 2011), notamment à l'arrosage nécessaire au maintien de la végétation. À Buckeye, cet usage représente un défi dans une ville en pleine croissance où s'installe une population venue d'ailleurs ainsi que le souligne la water conservation specialist de la Ville de Buckeye: « la plupart des gens ici viennent d'une autre région, du Canada notamment...Nous avons donc beaucoup de snowbirds ${ }^{19}$ qui achètent des résidences secondaires. Ils ont l'habitude d'utiliser l'eau de manière différente et ont des préférences paysagères différentes. Ils ne savent rien sur les plantes du désert, de quelle quantité d'eau elles ont besoin, etc. ${ }^{20} »$. La consommation d'eau pour le maintien de la végétation est l'une des cibles principales des politiques d'économies en eau en Arizona. L'État mais aussi les municipalités promeuvent depuis les années 1980 le xéropaysagisme qui requiert peu ou pas d'irrigation grâce à des plantes adaptées aux conditions arides. Le service municipal des eaux à Buckeye 
travaille dans cette direction mais se heurte aux intérêts des promoteurs immobiliers dont les produits standardisés, avec pelouses et terrains de golf, font leurs preuves depuis les années 1960 en Arizona. Par exemple, le service municipal des eaux a proposé au conseil municipal un décret qui interdit la pose de pelouse devant la maison-modèle des lotissements ce qui a suscité un tollé de la part des promoteurs: «j'ai essayé de les convaincre de ne pas mettre de pelouse devant la maison-modèle. Je leur ai dit : 'vous pourriez mettre une pelouse artificielle, comme ça ce serait bien vert quand même'. Mais ils n'ont pas voulu. Ensuite, je leur ai dit: 'vous allez mettre de la pelouse devant toutes les maisons mais il est possible que les gens n'aiment pas les pelouses !'. Ils ont dit qu'ils étaient sûrs de vendre les maisons avec de la pelouse et qu'ils étaient totalement opposés à toute restriction sur les pelouses. Et ils ont plus de pouvoir que moi... $\aleph^{21}$. Les promoteurs immobiliers évaluent la quantité d'eau qui sera utilisée par les résidents, mais ces coûts sont ensuite à la charge des résidents, inclus dans leur taxe foncière (CAGRD, 2014). D'après des forums en ligne qui discutent de la qualité de vie dans certains lotissements (comme Niche ou Yelp), les résidents sont peu au courant du fonctionnement de ce système, bien souvent la ligne de contribution au CAGRD indiquée sur leur déclaration d'impôts soulève des interrogations et la consommation de l'eau dans les parties communes (consommation excessive liée à l'arrosage notamment) peut susciter débat lors des réunions de copropriétaires...

À Phoenix, 80 \% des paysages urbains avaient de la pelouse dans les années 2000 alors que ce chiffre ne s'élève aujourd'hui qu'à $14 \%$, remplacés par des xériscapes (Robbins J., 2019). Un changement dans les attitudes à l'égard de l'environnement se met donc lentement en place dans les centres urbains d'Arizona, mais il s'opère dans le cadre d'une puissance publique locale forte, qui a mis des limites à la croissance à tout prix (souvent de fait, car l'espace n'est plus disponible). L'enjeu pour Buckeye réside donc surtout dans la faiblesse du pouvoir d'une structure municipale embryonnaire dans une ville qui se développe rapidement. La Ville se trouve donc confrontée à des problèmes de cohésion territoriale, alors qu'elle doit assurer non seulement le respect de la régulation environnementale en vigueur mais aussi la durabilité de son existence.

\section{Conclusion}

La trajectoire de Buckeye semble suivre le modèle de ces voisines d'Arizona centrale, Phoenix, Tempe, Mesa, Scottsdale : nées autour d'un canal d'irrigation construit à la fin du XIX siècle, elles sont désormais des municipalités de respectivement 1 million, 200000,500000 et 255000 habitants dans la cinquième plus grande aire urbaine des États-Unis. Le développement de Buckeye, qui repose en grande majorité sur la mobilisation des ressources en eau souterraine dans un contexte où ces dernières sont censées être régulées et protégées par le Groundwater Management Act, nous permet de mettre au jour la complexité et les ambivalences de la gestion de l'eau en Arizona. Si la croissance urbaine permet de transférer des usages agricoles de l'eau vers des usages urbains, elle exerce cependant une forte pression sur les ressources souterraines et met surtout en jeu le Central Arizona Groundwater Replenishment District. Chargé de réguler le niveau des nappes phréatiques grâce à un jeu de compensation et un système de renflouement des nappes phréatiques, le CAGRD a fini par fonctionner comme un mécanisme permettant aux propriétaires fonciers de démontrer la durabilité de leur approvisionnement et d'assurer ainsi la construction de nouveaux lotissements. Ce 
dispositif se trouve de plus en plus dépassé du fait de l'étalement urbain qui gagne des territoires de moins en moins pourvus en ressources en eau et de la menace de pénurie qui pèse sur l'eau du Colorado. On aurait pu penser que les efforts de gestion durable de l'eau mis en place dans un espace soumis à des problèmes de rareté permettraient de limiter la croissance urbaine mais ce n'est pas le cas. La gestion de l'eau est en fait déconnectée de la gestion foncière, ce qui représente un obstacle majeur au contrôle de la croissance à travers le facteur " eau ", pourtant déterminant. Si l'État d'Arizona se considère comme l'avant-garde de la gestion durable de l'eau aux États-Unis, encadrant des dispositifs à différentes échelles pour sécuriser et diversifier l'approvisionnement en eau, il est cependant peu regardant sur l'aspect gestion du territoire : de nombreux terrains sont encore disponibles - l'État est urbanisé à $25 \%$ - et le développement immobilier est un pilier important et prospère de l'économie régionale. Ainsi, l'eau est gérée dans le cadre d'un système administré et contrôlé par l'État d'Arizona, mais le foncier est mis en valeur et géré par les propriétaires sans vraie régulation de la part du gouvernement de l'État, ou local. Le rôle principal des acteurs publics en matière d'aménagement du territoire est même d'encourager l'aménagement en fournissant les infrastructures et services nécessaires comme les routes, les écoles, et bien sûr l'adduction en eau potable (Travis W., 2003; Gober P. et al., 2013). Ce mode de fonctionnement, où ce n'est pas la quantité d'eau disponible qui influence les modes de mise en valeur du territoire mais plutôt l'occupation des sols qui oriente la mise en valeur des ressources en eau, interroge sur la durabilité d'un modèle urbain hérité des années 1950 qui favorise l'étalement urbain, l'usage de la voiture, les piscines et les cours de golf dans le contexte de changement climatique.

\section{BIBLIOGRAPHIE}

AAWS Overview, « Arizona Department of Water Resources », 2019. https://new.azwater.gov/ aaws, page consultée le 20/02/2020

Abbott, Carl, The New Urban America: Growth and Politics in Sun Belt Cities, Chapel Hill, The University of North Carolina Press, 1987

Arnauld de Sartre, Xavier, Agriculture et changements globaux : Expertises globales et situations locales, Bern, Editions scientifiques internationales Peter Lang, 2016

Avery, Chris, Consoli, Carla, Glennon, Robert, et al., « Good intentions, unintented consequences: the Central Arizona Groundwater Replenishment District », Arizona Law Review, vol. 49, n 339, 2007

Barbier, Nicolas, « Vulnerability to water shortages in the 21st century's arid and semi-arid American West », L'Ordinaire des Amériques [En ligne], n²18, 2015. http://

journals.openedition.org/orda/1956, page consultée le 20/02/2020

Beau, Rémi et Larrère, Catherine, Penser l'anthropocène, Paris, Presses de Sciences Po, 2018

Benites-Gambirazio, Eliza, « The social logics of urban sprawl », in Poupeau, Franck et al. (dir.), Water Bankruptcy in the Land of Plenty, Boca Raton, CRC Press, 2016, p.121-140 
Benites-Gambirazio, Eliza, Coeurdray, Murielle et Poupeau, Franck, « Une promotion immobilière sous contraintes environnementales : les logiques sociales du périurbain dans les desert cities de l'Ouest états-unien », Revue Française de Sociologie, Vol. 57, n4, 2016, p. 735- 765

Berque, Augustin, Bonnin, Philippe et Ghorra-Gobin, Cynthia (dir.), La ville insoutenable, Paris, Belin, 2006

Bernat, Rebecca F.A., Megdal, Sharon B. et Eden, Susanna, « Long-Term Storage Credits: Analyzing Market-Based Transactions to Achieve Arizona Water Policy Objectives », Water, vol. $12, \mathrm{n}^{\circ} 2,2020$, p. 568

Blomquist, William A., Schlager, Edella et Heikkila, Tanya, « Common Water, Diverging Streams: Linking Institutions to Water Management in Arizona, California, and Colorado ", Washington, D.C., Resources for the Future, 2004

Bonneuil, Christophe et Fressoz, Jean-Baptiste, L'événement Anthropocène : la Terre, l'histoire et nous, Paris, Seuil, 2013

Boyer, Anne-Lise, Le Gouill, Claude, Poupeau, Franck, et al., « Conflit environnemental et participation publique dans les zones semi-arides de l'Ouest des États-Unis : le projet minier de Rosemont (comté de Pima, Arizona) », Participations, 2017, n³, p.189-217

Bruegmann, Robert, Sprawl: A compact history, Chicago, University of Chicago Press, 2006

CAGRD, « Your home is in the CARGD. What does that mean to you? ", 2014, http://cagrd.com/ documents/general-information/CAGRD_Brochure_2014_Final_2.pdf, page consultée le $20 / 02 / 2020$

CAGRD, Rates and Fees, 2019. https://www.cagrd.com/rates-and-fees, page consultée le $20 / 02 / 2020$

CAGRD, Plan of Operation, 2015. https://www.cagrd.com/documents/plan-of-operations/2015CAGRD-Plan-of-Operation.pdf, page consultée le 20/02/2020

Carrollo Engineers, City of Buckeye: Integrated Water Master Plan 2017, 2017. https:// www.buckeyeaz.gov/Home/ ShowDocument?id=2340.

Cayan, Daniel, Tyree, Mary, Kunkel, Kenneth, et al., « Future Climate: Projected Average », in Garfin et al., Assessment of Climate Change in the Southwest United States: A Report Prepared for the National Climate Assessment, A report by the Southwest Climate Alliance, Washington, D.C., Island Press, 2013, p.101-125

Cech, Thomas, Principles of Water Resources: History, Development, Management and Policy, Hoboken, Wiley, 2010

Clarke-Sather, Afton, Crow-Miller, Britt, Banister, Jeffrey M., et al., « The shifting geopolitics of water in the Anthropocene ", Geopolitics, vol. 22, n 2, 2017, p.332-359.

Coeurdray, Murielle, Poupeau, Franck, O’Neill, Brian et al., « Delivering more than water: The Salt River Project, the invention of an adaptative partnership of water management ", in Lorrain, Dominique et Poupeau, Franck (dir.), Water Regimes: Beyond the Public and Private Sector Debate, Abingdon, Routledge, 2016, p. 37-55

Colby, Bonnie et Jacobs, Katherine (dir.), Arizona Water Policy: Management Innovations in an Urbanizing Arid Region, Washington, D.C., Resources for the Future, 2007

Cortinas, Joan, Coeurdray, Murielle, O’Neill, Brian et al., « Les mégaprojets hydriques de l'ouest étasunien : histoire d'État(s) et gestion des ressources naturelles ", [Vertigo] La revue électronique en sciences de l'environnement, vol. 16, $\mathrm{n}^{\circ}$ 3, 2016. 
Crutzen, Paul J., « Geology of mankind-The Anthropocene », Nature, n 415, 2002, p. 23.

Euzen, Agathe et Morehouse, Barbara, « De l'abondance a la raison : Manières d'habiter à travers l'usage de l'eau dans une région semi-aride, l'exemple de Tucson en Arizona ", Norois [En ligne], $n^{\circ} 231$, 2014. http://journals.openedition.org/norois/5074, page consultée le 20/02/2020

Ferris, Kathleen et Porter, Sarah, « The Elusive Concept of an Assured Water Supply: The role of CAGRD and Replenishment ", Arizona State University, Morrison Institute, Kyl Center for Water Policy, 2019. https://morrisoninstitute.asu.edu/sites/default/files/ kyl_center_elusive_concept_101619.docx.pdf, page consultée le 20/02/2020

Gammage, Grady, Stigler, Monica, Daugherty, David et al., Watering the Sun Corridor, Managing Choices in Arizona's Megapolitan Area, Report, Arizona State University, Morrison Institute for Public Policy, Août 2011.

Gammage, Grady, The Future of the Suburban City: Lessons from Sustaining Phoenix, Washigton D.C., Island Press, 2016

Getches, David, "Constraints of Law and Policy on the Management of Western Water ", in Lewis, William (dir.), Water and Climate in the Western United States, Boulder, University Press of Colorado, 2003

Ghorra-Gobin, Cynthia, Villes et sociétés urbaines aux États-Unis, Paris, Armand Colin, Collection U, 2003

Gober, Patricia, Metropolitan Phoenix: Place Making and Community building in the Desert, Philadelphie, University of Pennsylvania Press, 2006

Gober, Patricia, Larson, Kelli, Quay, Ray et al. « Why land planners and water managers don't talk to one another and why they should! », Society \& Natural Resources, vol. 26, n³, 2013, p.356-364.

Heim, Carol, « Border Wars: Tax Revenues, Annexations and Urban Growth in Phoenix », International Journal of Urban and Regional Research, vol. 36, $\mathrm{n}^{\circ}$ 4, 2012, p. 831-859

Hirt, Paul, Snyder, Rachel, Hester, Cyrus et al., « Water Consumption and Sustainability in Arizona: A Tale of Two Cities ", Journal of the Southwest, vol. 59, n 1, 2017, p. 264-301

Jacobs, Katherine et Holway, James, « Managing for sustainability in an arid climate: lessons learned from 20 years of groundwater management in Arizona, USA », Hydrogeology Journal, vol. $12, \mathrm{n}^{\circ} 1,2004, \mathrm{p} .52-65$

Kenski, Hank, « Everything that grows needs water », in Melnick, Rob et Roepke, Deborah, Urban growth in Arizona: A policy analysis, Arizona State University, Morrison Institute for Public Policy, 1988

Lasserre, Frédéric, « L'Amérique a soif : Les besoins en eau de l'Ouest des États-Unis conduirontils Ottawa à céder l'eau du Canada ? ", Revue internationale d'Études Canadiennes, n 24, 2001, p.195214

Le Goix, Renaud, « Les communautés fermées dans les villes des États-Unis : aspects géographiques d'une sécession urbaine », L’Espace géographique, vol. 30, n¹, 2001, p. 81-93.

Le Goix, Renaud, « Les gated communities en Californie du Sud, un produit immobilier pas tout à fait comme les autres », L'Espace Géographique, vol. 31, nº 4, 2002, p.328-344.

Le Goix, Renaud, Sur le front de la métropole : une géographie suburbaine de Los Angeles, Paris, Editions de la Sorbonne, 2016 
Liddell, Eliane, «Phoenix : une métropole-oasis en péril ? ", L'Ordinaire des Amériques [En ligne], $n^{\circ} 218,2015$. http://journals.openedition.org/orda/1821, page consultée le 20/02/2020

Le Tourneau, François-Michel et Dubertret, Fabrice, « L'espace et l'eau, variables clés de la croissance urbaine dans le Sud-Ouest des États-Unis : le cas de Tucson et du Pima County (Arizona) ", L'Espace géographique, vol. 48, n 1, 2019, p.39-56.

Logan, Michael, Desert cities: the environmental history of Phoenix and Tucson, Pittsburgh, University of Pittsburgh Press, 2006

Matrix Design Group, Imagine Buckeye: 2040 General Plan, March 2018 http://

www.imaginebuckeye2040.com/images/docs/

buckeye_gp_final_draft_2018\%2003\%2014a_sm.pdf

Meck, Verlyne, Buckeye: Then and Now, Mount Pleasant, Arcadia Publishing, 2007

Megdal, Sharon B., " Arizona's Recharge and Recovery Programs » in Colby, Bonnie et Jacobs, Katharine, Arizona Water Policy: Management Innovations in an Urbanizing Arid Region, Washington, D.C., Resources for the Future, 2007, p.188-203

Miller, Carl, Water in the West: A High-Country News Reader, Corvallis, Oregon State University Press, 2000

Nédélec, Pascale, « Saisir l'étalement urbain dans un contexte états-unien : réflexions méthodologiques », Cybergeo, document 762, 2016. URL: https://journals.openedition.org/ cybergeo/27421, page consultée le 20/02/2020

Niraula, Rewati, Meixner, Thomas, Dominguez, Francina, et al., « How might recharge change under projected climate change in the western US? », Geophysical research letters, vol. 44, $\mathrm{n}^{\circ} 20$, 2017, p.10407-10418.

NOAA, « Local Climatological Data - Annual Summary for Phoenix, Arizona », 2018

O'Neill, Brian, Poupeau, Franck, Coeurdray, Murielle et al., « Laws of the River: Conflict and Cooperation on the Colorado River ", in Poupeau, Franck et al. (dir.), Water Bankruptcy in the Land of Plenty, Boca Raton, CRC Press, 2016

Parkman, Isaac, History of Buckeye Canal, Buckeye Irrigation Company, 1957

Poupeau, Franck, Gupta, Hoshin, Serrat-Capdevila, Aleix et al. (dir.), Water Bankruptcy in the Land of Plenty, Boca Raton, CRC Press, 2016

Purdue, Louise, « Construction, maintenance and abandonment of hydraulic systems: hydroclimatic or social constraints? A case study of prehistoric Hohokam irrigation systems (Phoenix, Arizona, USA)», Water History, Vol. 7, n 1, 2015, p.73-99.

Robbins, Jim, « In Era of Drought, Phoenix prepares for a future without Colorado River water », Yale Environment 360, 7 Février 2019. https://e360.yale.edu/features/how-phoenix-ispreparing-for-a-future-without-colorado-river-water? utm_campaign=coschedule\&utm_source=twitter\&utm_medium=americanrivers, page consultée le $20 / 02 / 2020$

Ross, Andrew, Bird on Fire: Lessons from the World's Least Sustainable City, Oxford, Oxford University Press, 2011

Schipper, Janine, Disappearing Desert: The Growth of Phoenix and the Culture of Sprawl, Norman, University of Oklahoma Press, 2008 
Sheridan, Thomas, « Arizona: The Political Ecology of a Desert State », Journal of Political Ecology, Vol. 2, $\mathrm{n}^{\circ} 1,1995, \mathrm{p} .41-57$

Sheridan, Thomas, Arizona: A History, Tucson, University of Arizona Press, 2012

Travis, William, « A changing geography: growth, land use, and water in the interior West » in Lewis, William (dir.), Water and Climate in the Western United States, Boulder, University Press of Colorado, 2003

Udall, Bradley et Overpeck, Jonathan, « The twenty-first century Colorado River hot drought and implications for the future », Water Resources Research, n 53, 2017, p.2404-2418. https:// agupubs.onlinelibrary.wiley.com/doi/full/10.1002/2016WR019638, page consultée le 20/02/2020 Wentz, Elizabeth et Gober, Patricia, « Determinants of Small-Area Water Consumption for the City of Phoenix, Arizona », Water Resources Management, vol. 21, n 11, 2007, p.1849-1863

Wright, Bruce, « Managing Water in Arizona: Making Tough Decisions », Ensuring Arizona's water quantity and quality into the 21st century, Report, Tucson, The University of Arizona, 1997

\section{NOTES}

1. Entre 2017 et 2018 , la ville de Buckeye a connu une croissance démographique de $+8,5 \%$. Voir "Fastest-Growing Cities primarily in the South and in the West", 23 mai 2019 : https:// www.census.gov/newsroom/press-releases/2019/subcounty-population-estimates.html

2. Notamment le Homestead Act de 1862 dans le cadre duquel les colons obtiennent des droits de propriété sur les terres publiques pour encourager leur mise en valeur, et le Desert Land Act de 1877 qui autorise l'accès aux terres fédérales pour leur mise en exploitation grâce à l'irrigation dans le but d'encourager le développement économique des terres arides et semi-arides de l'Ouest.

3. Les aires incorporées aux États-Unis sont des territoires qui ont reçu une charte de l'Etat pour accéder au statut de "ville" et qui sont gérés dès lors par une équipe municipale.

4. Entretien avec Jackie Meck par Verlyne Meck, 18 Février 2011, Arizona State Library, Archives and Public Records.

5. Le Salt River Project, instauré au début du XXe siècle, est le principal fournisseur en eau du centre de l'Arizona. Il gère huit réservoirs à l'est de Phoenix sur la rivière Salée et sur la rivière Verde et une dizaine de canaux qui approvisionnent la ville de Phoenix en eau.

6. Entretien avec Bruce Heiden, par Jean Denman, 21 Juin 2011, Arizona State Library, Archives and Public Records

7. Voir site Web du projet : https://douglasranchaz.com/

8. Organisation formée par les promoteurs immobiliers dans le but de gérer et commercialiser les maisons et les terrains d'un lotissement résidentiel. Toute personne achetant un logement dans un lotissement géré par une HOA doit en devenir membre, se conformer aux règlements en vigueur et en payer les charges.

9. Voir site Web de la Ville de Buckeye : https://www.buckeyeaz.gov/residents/water-resources 10. Selon le Plan America 2050 qui liste onze régions mégapolitaines. Voir: http:// www.america2050.org/arizona_sun_corridor.html

11. "These are the rational reasons that prospective residents, businesses and visitors should rank Buckeye high on their list of places to be. You can build here; we've got the land. We've got water, Buckeye sits on an aquifer (...) », p.22

12. « this time the cash crop became people rather than cotton, alfalfa or dairies ", p. 4 
13. Entretien, 5 juin 2019 : « When I came here, I thought that I would deal with people who don't want to see any changes here, with people who don't want anyone else to touch their lands...And no...We are in the exact opposite situation. Farmers want to make money...Today, kids don't really want to run the farm, developers are interested, so they want to sell their lands. And some of them won't say no to 30 million dollars you know... ».

14. Entretien avec un urbaniste de la Ville de Buckeye, 5 juin 2019 : « a nice rural environment »

15. Arizona Administrative Code, R12-15-724, Phoenix AMA Calculation of Groundwater Allowance and Extinguishment Credits

16. Entretien avec l'hydrologue de la Ville, 10 juillet 2018

17. Voir : "ASU Water Policy addresses new drought plan for state», Marshall Terrill, 27 août 2019 : http://morrisoninstitute.asu.edu/content/asu-water-policy-expert-addresses-newdrought-plan-state

18. D'après le Centre d'information sur l'eau : http://cieau.com/le-metier-de-leau/ressource-eneau-eau-potable-eaux-usees/quels-sont-les-usages-domestiques-de-leau/

19. Un snowbird est une personne qui migre des États du Nord des États-Unis et du Canada pour passer la saison d'hiver dans une résidence secondaire dans un État de la Sun Belt.

20. Entretien avec la water conservation specialist de la Ville de Buckeye, 10 juillet 2018 : « Most of the residents here are newcomers, they are from other areas, from Canada mostly...We have a lot of snowbirds out here, buying second homes. They're used to using water differently and have different landscaping you know. They don't know anything about desert plants, how much water they need and other things ».

21. Entretien avec l'hydrologue de la Ville de Buckeye, 10 juillet 2018 : « And I tried to convince them that they could not put turf for the model-home. I said "you could put artificial turf there, that still looks green". But they didn't want to do that. But then I said 'well, if you put in turf what if someone doesn't want it ?' but then they said that they are $100 \%$ sure to sell them later with turf, that they are opposed to any restriction on turf... And they have more power than I do ».

\section{RÉSUMÉS}

La ville de Buckeye, en bordure de l'aire urbaine de Phoenix, la capitale de l'État d'Arizona, est d'après le recensement américain, la ville qui croit le plus vite des États-Unis. Située dans un environnement semi-aride, la ville est née au bord d'un canal d'irrigation dans le contexte de la Conquête de l'Ouest. De petite localité agricole, elle se transforme en banlieue (suburb) attractive de Phoenix, où se multiplient les masterplanned communities, enclaves et oasis résidentielles. Ce développement urbain repose en grande majorité sur l'exploitation des nappes phréatiques, contrôlée depuis les années 1980 par le Groundwater Management Act, une loi de l'État d'Arizona, considérée comme à l'avant-garde de la gestion durable de l'eau. A travers le cas de Buckeye, cet article explore les modalités de la gestion de l'eau à l'échelle municipale et en montre les contradictions. En effet, si un certain nombre de dispositifs et garde-fous ont été mis en place dans le but de réguler l'exploitation de la ressource, le laisser-faire des divers échelons de la puissance publique (État, municipalités) concernant le foncier n'aide pas à soulager la pression sur la ressource qui pourtant se raréfie dans le contexte du changement climatique. 
The city of Buckeye, on the west side of the Phoenix urban area (the state capital of Arizona) is the fastest growing city in the United States. Located in a semi-arid environment, the city was born at the edge of an irrigation canal in the context of the Conquest of the West. From a small farming community, Buckeye has transformed into an attractive suburb of Phoenix composed of multiple oasis-like masterplanned communities. However, this urban development is largely based on the exploitation of groundwater, regulated since the 1980s by the Groundwater Management Act, a law passed by the State of Arizona considered to be at the forefront of sustainable water management. This paper builds on how water is managed at the municipal level in Buckeye to explore the contradictions of water management in Arizona. Indeed, numbers of measures and safeguards were put in place in order to regulate the exploitation of the resource. The laissezfaire attitude of the various levels of government (State, municipalities) concerning land management is not helping to lower the pressure on the resource, which is becoming increasingly rare in the context of climate change.

La ciudad de Buckeye, que limita con el área urbana de Phoenix, la capital del estado de Arizona, es la ciudad con mayor crecimiento en los Estados Unidos. Ubicada en un clima semiárido, la ciudad nació al borde de un canal de riego durante la Conquista del Oeste. De una pequeña comunidad agrícola, se transformó en una periferia atractiva de Phoenix compuesta de múltiples comunidades planificadas como oasis. Sin embargo, este desarrollo urbano se basa en gran medida en la explotación de aguas subterráneas, controlada desde la década de 1980 por la Ley de Gestión de Aguas Subterráneas, una ley del Estado de Arizona, considerada a la vanguardia de la gestión sostenible del agua. A través del caso de Buckeye, este artículo explora las modalidades de gestión del agua a nivel municipal y muestra las contradicciones de la gestión del agua en Arizona. De hecho, si se estableciera un cierto número de dispositivos y salvaguardas para regular la explotación del recurso, la debilidad de los diversos niveles del poder público (Estado, municipios) con respecto a la tierra no hace nada para aliviar la presión sobre el recurso, que se está volviendo cada vez más raro en el contexto del cambio climático.

\section{INDEX}

Mots-clés : Gestion de l'eau, étalement urbain, nappe phréatique, environnement semi-aride, États-Unis

Palabras claves : Gestión del agua, expansión urbana, aguas subterráneas, región semiárida, Estados Unidos

Keywords : Water management, urban sprawl, groundwater, semi-arid land, United States

\section{AUTEURS}

\section{ANNE-LISE BOYER}

Anne-Lise Boyer est doctorante en géographie au laboratoire Environnement Ville Société. UMR 5600, ENS de Lyon. annelise.boyer@ens-lyon.fr

\section{REBECCA F.A. BERNAT}

Rebecca F.A. Bernat est doctorante à l'Université d'Arizona. Department of Environmental Science, College of Agriculture and Life Sciences rebeccabernat@email.arizona.edu 